\title{
Overlap between nutrient metabolism and chemotherapy detoxification in triple negative breast cancer
}

\author{
G. Cioccoloni ${ }^{1}$, A. Websdale ${ }^{1}$, C. Soteriou ${ }^{1}$, P. Lianto ${ }^{1}$, S.A. Hutchinson ${ }^{2}$, T.A. Hughes ${ }^{3}$, \\ E.J.R. Vasconcelos ${ }^{4}$, N. Forde ${ }^{3,4}$, B. Kim ${ }^{5}$, M.A. Zulyniak ${ }^{1}$ and J.L. Thorne ${ }^{1}$ \\ ${ }^{1}$ School of Food Science and Nutrition, University of Leeds, Leeds, UK, \\ ${ }^{2}$ Division of Cancer Therapeutics, Institute of Cancer Research, Chester Beatty Laboratories, London, UK, \\ ${ }^{3}$ School of Medicine, University of Leeds, Leeds, UK, \\ ${ }^{4}$ LeedsOmics, FMH/FBS, University of Leeds, Leeds, UK and \\ ${ }^{5}$ Leeds teaching Hospitals NHS Trust, Leeds, UK
}

Detoxification and nutrient metabolism are connected in healthy tissues to prevent toxic accumulation of metabolic intermediates and end-products $^{(1)}$. These pathways also enhance processing of xenobiotics such as anti-cancer drugs ${ }^{(2)}$. Resistance to anti-cancer drugs remains a major barrier to successful treatment of many cancer types, yet clinical tools that predict which treatment regimen would be most effective at individual patient level remain underdeveloped. Nutrient regulated pathways have not been evaluated as predictors of chemotherapy resistance previously. We aimed to identify the intersection between chemoresistance biomarkers (CR-biomarkers) and nutrient dependent transcription factors (nutrient-TFs) to better understand how nutrition and anti-cancer drug pathways interact at the molecular level.

First, to identify CR-biomarkers we performed a systematic literature review (PROSPERO \#CRD42021243047) to identify genes that confer resistance to drugs commonly used to treat the triple negative subtype of breast cancer (TNBC). After duplicates were removed, 816 abstracts were each screened by two independent researchers. Full text analysis was performed on 149 studies, this identifed 144 CR-biomarkers, of which 64 were validated in both clinical studies and with a described molecular mechanism of action. mRNA expression of these 64 CR-biomarkers and 36 nutrient-TFs from the tumours of 307 TNBC patients were obtained from METABRIC ${ }^{(3,4)}$ using cBioportal.org. Patients were split into those who relapsed/died (183) or those who were disease-free at census (124). Pair-wise correlation analyses between nutrient-TF (transcription factor) and CR-biomarker (potential target genes) were performed using two-tailed non-parametric Spearman correlation analysis. High stringency significantly correlated gene pairs (q-value $<0.01$ ) were retained and plotted as networks (Cytoscape v3.8.2). The promoters of CR-biomarkers were assessed for putative (sequence analysis using TRANSFAC) and validated (ChIP-sequencing; Cistrome.org) binding by any nutrient-TFs with which they significantly correlated at the expression level.

From correlation analyses, three sub-clusters were identified: i) cholesterol and oxysterol transcription factors, where LXR $\alpha$ was mostly positively correlated with CR-biomarkers, and $\operatorname{LXR} \beta, \operatorname{ROR} \gamma$ and $\operatorname{ROR} \alpha$ receptors are mostly inversely correlated with CR-biomarkers $(q<0.01)$; ii) fatty acid transcription factors PPAR $\alpha$ and PPAR $\delta$ positively correlated with CR-biomarkers, whilst PPAR $\gamma$ negatively correlated with multiple CR-biomarkers $(\mathrm{q}<0.01)$; iii) retinoid transcription factors $\mathrm{RAR} \alpha$ and $\mathrm{RXR} \alpha$ were almost exclusively inversely correlated with CR-biomarkers $(\mathrm{q}<0.01)$. Promoter analysis revealed that $\mathrm{LXR} \alpha, \mathrm{PPAR} \alpha$ and $\mathrm{RXR} \alpha$ bound to the regulatory regions of CR-biomarkers with which they correlated at mRNA expression level. Specifically, we have identified with high confidence, CR-biomarkers under the control of LXR $\alpha$ (TAZ, CD274, ALDH2, MAPK1, TRPM2, EP300), PPAR $\alpha$ (EGFR, RAD21 and RAD51), and RXR $\alpha$ (MYC, TOP2A, RIPK2 and TPX2).

These results suggest personalized nutritional interventions may improve TNBC patient outcomes, and that it is timely to evaluate whether a patient's adherence to the World Cancer Research Fund cancer prevention recommendations is a predictor of tumour response to chemotherapy.

\section{References}

1. Lee N, Spears ME, Carlisle AE, et al. (2020) Oncogene 39, 5709-5720.

2. Hutchinson SA, Websdale A, Cioccoloni G, et al. (2021) Oncogene (In Press).

3. Curtis C, Shah SP, Chin SF, et al. (2012) Nature 486, 346-52.

4. Pereira B, Chin SF, Rueda OM, et al. (2016) Nat Commun 7, 11479. 